Geometry 83 Topology Monographs

Volume 1: The Epstein birthday schrift

Pages 295-301

\title{
On the fixed-point set of automorphisms of non-orientable surfaces without boundary
}

\author{
M IZQUiERDo \\ D Singerman
}

\begin{abstract}
Macbeath gave a formula for the number of fixed points for each non-identity element of a cyclic group of automorphisms of a compact Riemann surface in terms of the universal covering transformation group of the cyclic group. We observe that this formula generalizes to determine the fixed-point set of each non-identity element of a cyclic group of automorphisms acting on a closed non-orientable surface with one exception; namely, when this element has order 2. In this case the fixed-point set may have simple closed curves (called ovals) as well as fixed points. In this note we extend Macbeath's results to include the number of ovals and also determine whether they are twisted or not.
\end{abstract}

AMS Classification 20F10, 30F10; 30F35, 51M10, 14H99

Keywords Automorphism of a surface, NEC group, universal covering transformation group, oval, fixed-point set

For David Epstein on the occasion of his sixtieth birthday

\section{Introduction}

Let $Y$ be a compact non-orientable Klein surface of genus $p \geq 3$. By genus here we mean the number of cross-caps of the surface. Let $t: Y \rightarrow Y$ be an automorphism of order $M$. If $1 \leq i<M$ and if $i \neq M / 2$ then the fixed-point set of $t^{i}$ consists of isolated fixed points and their number can be calculated, as described below, by a formula which is completely analogous to Macbeath's formula [5] concerning automorphisms of Riemann surfaces. However, if $M=$ $2 N$ then the fixed-point set of the involution $t^{N}$ consists of a finite number $n$ of disjoint simple closed curves called ovals together with a finite number of isolated fixed points [2], [6]. The ovals may be twisted or untwisted which means that they have Möbius band or annular neigbourhoods respectively. 
In this note we calculate the number of ovals and isolated fixed-points of $t^{N}$ and whether the ovals are twisted or not.

The information is given, as in Macbeath [5] in terms of the universal covering transformation group.

The authors acknowledge Mälardalen University and the Swedish Natural Science Research Council for financial support.

\section{The universal covering transformation group}

If $Y$ is a compact non-orientable Klein surface of genus $p \geq 3$ then the orientable two-sheeted covering surface of $Y$ has genus $\geq 2$, so that the universal covering space of $Y$ is the upper half-plane $\mathrm{H}$ (with the hyperbolic metric) and the group of covering transformations is a non-orientable surface subgroup $K$ generated by glide-reflections. If $G$ is a group of automorphisms of $Y$ then the elements of $G$ lift to a non-euclidean crystallographic (NEC) group $\Gamma$ acting on $H$. There is a smooth epimorphism

$$
\theta: \Gamma \rightarrow G
$$

whose kernel is $K$, where smooth means that $\theta$ preserves the orders of elements of finite order in $\Gamma$. The transformation group $(\Gamma, \mathcal{H})$ is called the universal covering transformation group of $(G, Y)$.

Now let $G=\left\langle t \mid t^{2 N}=1\right\rangle$ be a cyclic group of order $2 N$. As $\theta$ is smooth we must have $\theta(c)=t^{N}$ for every reflection $c$ in $\Gamma$. Also we cannot have two distinct reflections in $\Gamma$ whose product has finite order. So it follows, in the canonical presentation of NEC groups as given in [4] or [3], that $\Gamma$ has empty period cycles.

Thus $\Gamma$ has signature of the form

$$
s(\Gamma)=\left(g ; \pm ;\left[m_{1}, \ldots, m_{n}\right] ;\left\{(\quad)^{k}\right\}\right)
$$

with $k$ empty period cycles; then $\Gamma$ has one of the two presentations depending on whether there is a + or a - in the signature;

for the $(+)$ case

$$
\begin{array}{r}
x_{1}, \ldots, x_{n}, e_{1}, \ldots, e_{k}, c_{1}, \ldots, c_{k}, a_{1}, b_{1}, \ldots, a_{g}, b_{g} \\
x_{i}^{m_{i}}=1, i=1, \ldots, n, c_{j}^{2}=c_{j} e_{j}^{-1} c_{j} e_{j}=1, j=1, \ldots, k, \\
x_{1} \ldots x_{n} e_{1} \ldots e_{k} a_{1} b_{1} a_{1}^{-1} b_{1}^{-1} \ldots a_{g} b_{g} a g h^{-1} b_{g}^{-1}
\end{array}
$$


for the $(-)$ case

$$
\begin{array}{r}
x_{1}, \ldots, x_{n}, e_{1}, \ldots, e_{k}, c_{1}, \ldots, c_{k}, d_{1}, \ldots, d_{g} \\
x_{i}^{m_{i}}=1, i=1, \ldots, n, c_{j}^{2}=c_{j} e_{j}^{-1} c_{j} e_{j}=1, j=1, \ldots, k, x_{1} \ldots x_{n} e_{1} \ldots e_{k} d_{1}^{2} \ldots d_{g}^{2}
\end{array}
$$

In these presentations the generators $x_{i}$ are elliptic elements, the generators $c_{j}$ are reflections, the generating reflections of $\Gamma$, and the generators $e_{j}$ are orientation-preserving transformations called the connecting generators. Each empty period cycle corresponds to a conjugacy class of reflections in $\Gamma$.

One important fact to note about these presentations is that the connecting generator $e_{j}$ commutes with the generating reflection $c_{j}$, and in fact the centralizer of $c_{j}$ in $\Gamma$ is just the group $g p\left\langle c_{j}, e_{j}\right\rangle \cong C_{2} \times C_{\infty}$. (See [8])

\section{The fixed-point set of a power of $t$}

Let $Y$ be a non-orientable surface of topological genus $p \geq 3$ and let $t$ be an automorphism of order $2 N$. If $1 \leq i<2 N$ and $i \neq N$ then the number of fixed points of the automorphism $t^{i}$ is given by Macbeath's formula (see [5] ). If $t^{i}$ has order $d$ than $t^{i}$ has

$$
2 N \sum_{d \mid m_{j}} \frac{1}{m_{j}}
$$

fixed points, where $m_{j}$ runs over the periods in $s(\Gamma)$.

This is because Macbeath's proof (applying to Fuchsian groups) only uses the facts that each period corresponds to a unique conjugacy class of elliptic elements of $\Gamma$, and each elliptic element has a unique fixed point in $\mathrm{H}$. Now, the number of isolated fixed points of $t^{i}$ is independent of the smooth epimorphism $\theta$ above. However the epimorphism $\theta$ does play a part in the number of ovals of $t^{N}$.

Theorem 3.1 Let $Y$ be a non-orientable surface of topological genus $p \geq 3$. Let $G \cong C_{2 N}=\left\langle t \mid t^{2 N}=1\right\rangle$ be a group of automorphisms of $Y$, and let $\theta$ and $\Gamma$ be as described in equations 1 and 2. If $\theta\left(e_{j}\right)=t^{v_{j}}$ than the number of ovals of the involution $t^{N}$ is

$$
\sum_{j=1}^{k}\left(N, v_{j}\right)
$$

and the number of isolated fixed points of $t^{N}$ is

$$
2 N \sum_{m_{j} \text { even }} \frac{1}{m_{j}} .
$$


Proof Let $\Lambda=\theta^{-1}\left(\left\langle t^{N}\right\rangle\right)$ so that $\Lambda$ contains the group $K=K \operatorname{er} \theta$ with index 2 . Now, $\Lambda$ must have signature of the form

$$
s(\Gamma)=\left(g ; \pm ;\left[2^{(r)}\right] ;\left\{(\quad)^{s}\right\}\right)
$$

with $r$ periods equal to 2 and $s$ empty period cycles.

The reason that all periods in $\Lambda$ are equal to 2 is because if $m_{j}$ in $s(\Gamma)$ is even then $x_{j}^{m_{j} / 2} \in \Lambda$ and any elliptic element of $\Lambda$ are conjugate to some $x_{j}^{m_{j} / 2}$ (see [7] ).

By results in [2] (see also [3]), $\mathrm{r}$ is the number of isolated fixed points of $t^{N}$ and is given by Macbeath's formula

$$
2 N \sum_{m_{j} \text { even }} \frac{1}{m_{j}}
$$

It also follows from [2] that the number of ovals of $t^{N}$ is just the number $s$ of period cycles in $\Lambda$, which corresponds to the number of conjugacy classes of reflections in $\Lambda$. As a reflection $c_{j}$ in $\Lambda$ belongs also to $\Gamma$ and the group $\Gamma$ has $k$ conjugacy classes of reflections, we just have to determine into how many $\Lambda$-conjugacy classes the $\Gamma$-conjugacy class of $c_{j}$ splits. We shall use the epimorphism $\theta$ to calculate this number.

There is a transitive action of $\Gamma$ on the $\Lambda$-conjugacy classes of $c_{j}$ in $\Lambda$ by letting $\gamma \in \Gamma$ map the reflection $g c_{j} g^{-1}$ to $g \gamma c_{j} \gamma^{-1} g^{-1}$, with $g \in \Lambda$. (Because $\Lambda \triangleleft \Gamma)$. Clearly, if $\lambda \in \Lambda$ then $\lambda$ has a trivial action on these $\Lambda$-conjugacy classes. So we have an action of $\Gamma / \Lambda \cong C_{2 N} / C_{2} \cong C_{N}$ on these classes. As the centralizer of $c_{j}$ in $\Gamma$ is just $\left\langle c_{j}, e_{j}\right\rangle$, the stabilizer of the $\Lambda$-conjugacy classes of $c_{j}$ in $\Lambda$ are the cosets $\Lambda, \Lambda e_{j}, \ldots, \Lambda e_{j}^{\delta_{j}-1}$, where $\delta_{j}=\exp _{\Lambda} e_{j}$, the least positive power of $e_{j}$ that belongs to $\Lambda$. Now, let $\varepsilon_{j}=\exp _{K} e_{j}$. Then either $\varepsilon_{j}=\delta_{j}$ or $\varepsilon_{j}=2 \delta_{j}$.

The additive group $Z_{2 N}$ contains a subgroup isomorphic to $Z_{N}$ and $a \in Z_{N}$ has order $\frac{N}{(N, a)}$ in $Z_{N}$ so that $a$ has the same order in $Z_{2 N}$ if and only if $(2 N, a)=2(N, a)$. If $(2 N, a)=(N, a)$ then the order of $a$ in $Z_{2 N}$ is twice the order of $a$ in $Z_{N}$ and we then find that

$$
\varepsilon_{j}=\delta_{j} \quad \text { if } \quad\left(2 N, v_{j}\right)=2\left(N, v_{j}\right)
$$

and

$$
\varepsilon_{j}=2 \delta_{j} \quad \text { if } \quad\left(2 N, v_{j}\right)=\left(N, v_{j}\right),
$$

where $\theta\left(e_{j}\right)=t^{v_{j}}$. 
By the above argument on the action of $\Gamma / \Lambda$ on the $\Lambda$-conjugacy classes of $c_{j}$ we see that the number of such classes is $N / \delta_{j}$, which is if $\varepsilon_{j}=\delta_{j}$

$$
\frac{N}{\delta_{j}}=\frac{N}{\varepsilon_{j}}=\frac{N\left(2 N, v_{j}\right)}{2 N}=\frac{\left(2 N, v_{j}\right)}{2}=\left(N, v_{j}\right)
$$

or if $\varepsilon_{j}=2 \delta_{j}$

$$
\frac{N}{\delta_{j}}=\frac{2 N}{\varepsilon_{j}}=\frac{2 N\left(2 N, v_{j}\right)}{2 N}=\left(2 N, v_{j}\right)=\left(N, v_{j}\right)
$$

Thus in both cases the generating reflection $c_{j}$ of $\Gamma$ induces $\left(N, v_{j}\right)$ conjugacy classes of reflections in $\Lambda$. Thus the number of ovals of $t^{N}$ in $Y$ is

$$
\sum_{j=1}^{k}\left(N, v_{j}\right)
$$

Theorem 3.2 The ovals of $t^{N}$ in $Y$ induced by the $j$ th period cycle in $\Gamma$ are twisted if $\left(2 N, v_{j}\right)=\left(N, v_{j}\right)$ and untwisted if $\left(2 N, v_{j}\right)=2\left(N, v_{j}\right)$.

Proof As we have found in Theorem 3.1, the $j$ th empty period cycle in $\Gamma$ induces $\left(N, v_{j}\right)$ empty period cycles in $\Lambda$. The generating reflections of these period cycles are just conjugates of $c_{j}$ in $\Gamma$ and, as the corresponding connecting generator $e_{j}$ is just the orientation-preserving element generating the centralizer of $c_{j}$ in $\Gamma$, we see that the connecting generator of each of the period cycles in $\Lambda$ induced by the $j$ th period cycle in $\Gamma$ is just conjugate to $e_{j}^{\delta_{j}}, \delta_{j}=\exp _{\Lambda} e_{j}$ as in the proof of Theorem 3.1. Now, let $\theta^{\prime}: \Lambda \rightarrow C_{2}=g p\langle\xi\rangle$, where $\xi=t^{N}$, be the restriction of the epimorphism $\theta: \Gamma \rightarrow C_{2 N}$. Then

if $\varepsilon_{j}=\delta_{j}$

$$
\theta^{\prime}\left(e_{j}^{\delta_{j}}\right)=\theta^{\prime}\left(e_{j}^{\varepsilon_{j}}\right)=\theta\left(e_{j}^{\varepsilon_{j}}\right)=1
$$

if $\varepsilon_{j}=2 \delta_{j}$

$$
\theta^{\prime}\left(e_{j}^{\delta_{j}}\right)=\theta^{\prime}\left(e_{j}^{\frac{\varepsilon_{j}}{2}}\right)=\theta\left(e_{j}^{\frac{\varepsilon_{j}}{2}}\right)=\xi
$$

$\xi$ the generator of $C_{2}$. Generally, if $c$ is the generating reflection of an empty period cycle of $\Lambda$ and $e$ is the corresponding connecting generator then figures 1 and 2 show that $\theta^{\prime}(e)=1$ corresponds to an untwisted oval while $\theta^{\prime}(e)=\xi$ corresponds to a twisted oval.

However, as in the proof of Theorem $3.1 \varepsilon_{j}=\delta_{j}$ if and only if $\left(2 N, v_{j}\right)=$ $2\left(N, v_{j}\right)$ and hence we have untwisted ovals while $\varepsilon_{j}=2 \delta_{j}$ if and only if $\left(2 N, v_{j}\right)=\left(N, v_{j}\right)$ and we have twisted ovals. 


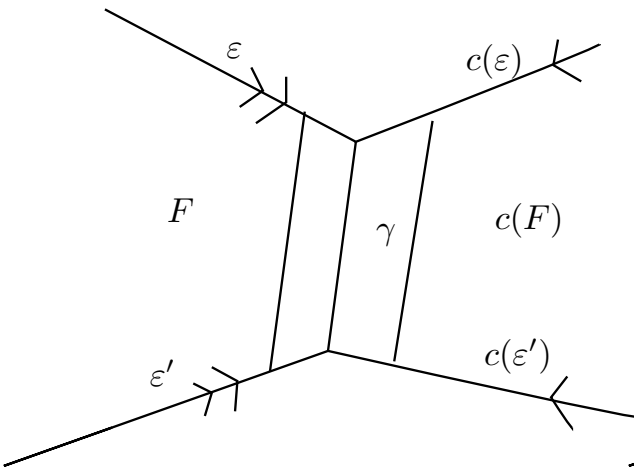

Figure 1: $\theta^{\prime}(e)=1$ so $e \in K$

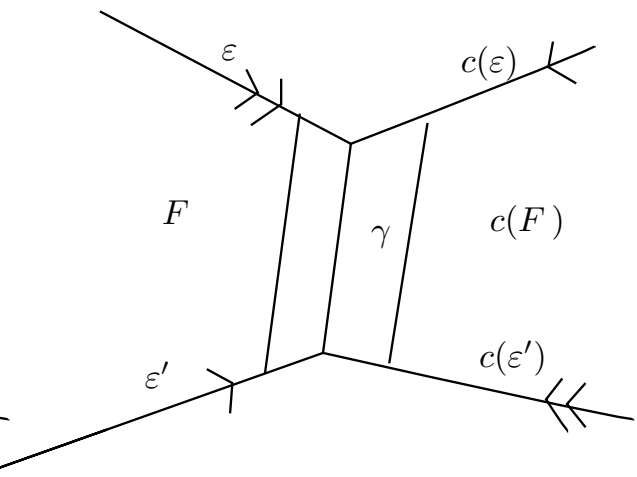

Figure 2: $\quad \theta^{\prime}(e)=\xi$ so $c e \in K$

\section{Bounds and examples}

In [6] (also see [2]) Scherrer showed that that if an involution of a non-orientable surface of genus $p$ has $|F|$ fixed points and $|V|$ ovals then

$$
|F|+2|V| \leq p+2 .
$$

In our examples we will show that for any integer $N$ we can find a non-orientable surface of genus $p$ admitting a $C_{2 N}$ action with generator $t$ such that $t^{N}$ attains the Scherrer bound.

Example 1 Bujalance [1] found the maximum order for an automorphism $t$ of a non-orientable surface $Y$ of genus $p \geq 3$; it is $2 p$ for odd $p$ and $2(p-1)$ for even $p$. The universal covering transformation group $\Gamma$ has signature $s(\Gamma)=$ $(0 ;[2, p] ;\{(\quad)\})$ for odd $p$, and signature $s(\Gamma)=(0 ;[2,2(p-1)] ;\{(\quad)\})$ for even $p$. There is, essentially, only one way of defining the epimorphism $\theta$ in each case:

if $p$ is odd, we define $\theta: \Gamma \rightarrow C_{2 p}$ by $\theta\left(x_{1}\right)=t^{p}, \theta\left(x_{2}\right)=t^{2}, \theta(c)=t^{p}$, and $\theta(e)=t^{p-2}$,

if $p$ is even, we define $\theta: \Gamma \rightarrow C_{2(p-1)}$ by $\theta\left(x_{1}\right)=t^{p-1}, \theta\left(x_{2}\right)=t^{1}, \theta(c)=t^{p-1}$, and $\theta(e)=t^{p-2}$.

Using Macbeath's formula (5) we see that the involution $t^{p}$ has $p$ fixed points for surfaces of both odd and even genera. Now, if $p$ is odd then the involution $t^{p}$ also has, by Theorems 3.1 and 3.2, one twisted oval if $p$ is odd as $(p, p-2)=$ $(2 p, p-2)=1$. If $p$ is even then the involution $t^{p-1}$ has, by Theorems 3.1 and 3.2 , one untwisted oval as $(p-1, p-2)=1$ and $(2(p-1), p-2)=2(p, p-2)=2$. We note that the involution $t^{p}$ obeys the Scherrer bound. Note that the orders 
of the cyclic groups in Bujulance's examples are $\equiv 2 \bmod 4$. Our second example shows that the Scherrer bound can be obtained for the involution in a $C_{4}$ action.

Example 2 Let $Y$ be a non-orientable surface of genus $p \geq 3$, and let $t$ be an automorphism of $Y$ of order 4 . Let $\Gamma$ have signature

$$
\left(0 ;+;\left[2^{(r)}, 4,4\right] ;(\quad)^{k}\right)
$$

and define a smooth epimorphism $\theta: \Gamma \rightarrow C_{4}$ by mapping the generators of order two to $t^{2}$, the two generators of order 4 to $t$ and $t^{-1}$ and the connecting generators to the identity. We then find that for the involution $t^{2},|F|=2 r+2$, and $|V|=2 k$, and $p=4 k+2 r$, so that we find infinitely many surfaces where the Scherrer bound is attained for the involution in $C_{4}$. This is easily extended to groups of order $4 m$ by replacing the two periods 4 in the signature of $\Gamma$ by $4 m$.

\section{References}

[1] E Bujalance, Cyclic groups of automorphisms of compact non-orientable Klein surfaces without boundary, Pac. J. of Math. 109 (1983) 279-289

[2] E Bujalance, A F Costa, S Natanzon, D Singerman, Involutions of compact Klein surfaces, Math. Z. 211 (1992) 461-478

[3] E Bujalance, J J Etayo, J M Gamboa, G Gromadzki, A combinatorial approach to groups of automorphisms of bordered Klein surfaces, Lect. Notes in Math. vol. 1439, Springer-Verlag (1990)

[4] A M Macbeath, The classification of non-euclidean plane crystallographic groups, Canad. J. Math. 19 (1967) 1192-1205

[5] A M Macbeath, Action of automorphisms of a compact Riemann surface on the first homology group, Bull. London. Math. Soc. 5 (1973) 103-108

[6] W Scherrer, Zur Theorie der endlichen Gruppen topologischer Abbildungen von geschlossenen Flächen in sich, Comment. Math. Helv. 1 (1929) 60-119

[7] D Singerman, Subgroups of Fuchsian groups and finite permutations groups, Bull. London. Math. Soc. 2 (1970) 319-323

[8] D Singerman, On the structure of non-euclidean crystallographic groups, Proc. Camb. Phil. Soc. 76 (1974) 233-240

Department of Maths, Mälardalen University, 72123 Västerås, Sweden

and

Department of Maths, University of Southampton, Southampton, SO17 1BJ, UK

Email: mio@mdh.se, ds@maths.soton.ac.uk

Received: 15 November 1997 